\title{
Increasing the dimensionality of cryogenic molecular coolers: Gd-based polymers and metal-organic frameworks
}

\author{
Giulia Lorusso, ${ }^{a}$ Maria A. Palacios, ${ }^{b}$ Gary S. Nichol, ${ }^{b}$ Euan K. Brechin, ${ }^{b}$ Olivier Roubeau* ${ }^{a}$ and Marco \\ Evangelisti*a
}

\author{
${ }_{5}$ Received (in $\left.X X X, X X X\right)$ Xth $X X X X X X X X X 20 X X$, Accepted Xth $X X X X X X X X X 20 X X$ \\ DOI: $10.1039 / b 000000 x$
}

The magnetothermal properties of a coordination polymer and a metal-organic framework (MOF) based on $\mathrm{Gd}^{3+}$ ions are reported. An equally large cryogenic magnetocaloric 10 effect (MCE) is found, irrespective of the dimensionality. This combined with their robustness makes them appealing for widespread magnetic refrigeration applications.

Gadolinium-based molecular magnetism and cryogenic magnetic refrigeration are closely interrelated topics that have received a 15 rejuvenated $^{1}$ and increased interest in the recent literature. ${ }^{2-7}$ The driving force stems from the experimental observation that the magnetocaloric effect (MCE) of this class of materials can outperform that of well-established magnetic refrigerants at liquid-helium temperatures. MCE describes the changes of 20 magnetic entropy and adiabatic temperature, following a change of the applied magnetic field, and can be exploited for magnetic refrigeration in a process known as adiabatic demagnetization. ${ }^{8}$ $\mathrm{Gd}^{3+}$ is a common constituent element for molecular refrigerant materials, because large MCE can be favoured by: ${ }^{2}$ (a) its ${ }^{8} S_{7 / 2}$ 25 ground state, which provides the largest entropy per single ion, (b) its quenched orbital momentum, which implies that crystal field effects are extremely small, and (c) its weak superexchange interactions, which result in low-lying excited spin states. For Gd-metal and most high- $T$ solid-state refrigerant materials, the 30 MCE is driven by the mechanism of magnetic ordering. ${ }^{8}$ For Gdbased molecular refrigerants, thermal fluctuations are typically stronger than that of magnetic origin between molecules, unless experiments are carried out deep in the sub-kelvin regime. ${ }^{7}$ Therefore for such materials, one would expect the magnetic 35 dimensionality to play no dominant role in the MCE. So far, MCE investigations have focused on high-spin OD Gd clusters, ${ }^{1,4,7}$ with rare exceptions. ${ }^{5,6}$ Here, we report the MCE of two extended networks of $\mathrm{Gd}^{3+}$ ions with 2D and 3D topologies, respectively the novel $\left[\mathrm{Gd}(\mathrm{HCOO})(\mathrm{OAc})_{2}\left(\mathrm{H}_{2} \mathrm{O}\right)_{2}\right]$ (1) and the 40 previously reported MOF $\left[\mathrm{Gd}_{2}(\mathrm{~N}-\mathrm{BDC})_{3}(\mathrm{dmf})_{4}\right](2, \mathrm{~N}-\mathrm{BDC}=2-$ amino-1,4-benzenedicarboxylic acid). ${ }^{9}$ Their MCE are indeed found qualitatively similar to that of the best Gd-based clusters. ${ }^{4}$ Providing a more robust, 3D framework structure to molecular coolers could facilitate their widespread applications through

45 synthetic and technological strategies already developed in MOF science. ${ }^{10}$ Our MCE results for 2 nicely complement those already reported for other MOF materials, viz. Prussian blue analogues, investigated for magnetic refrigeration at high temperatures. $^{11}$
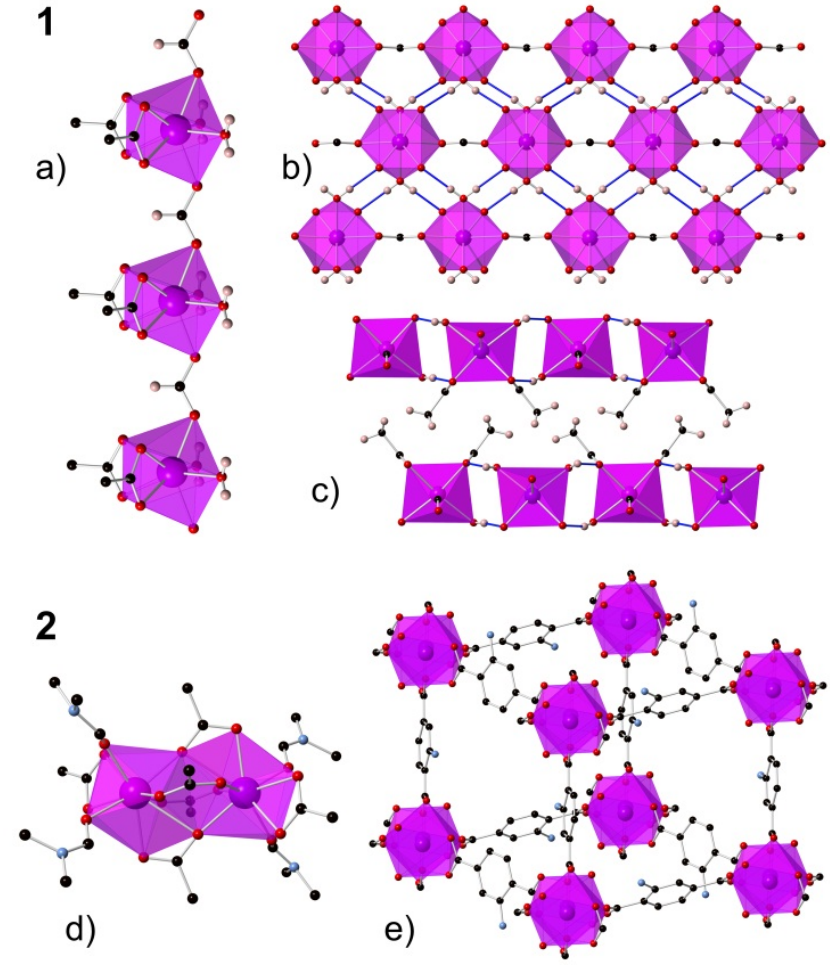

Fig. 1 Top: views of the structure of 1: a) coordination chains propagating along the $b$ axis; b) chains H-bonded into sheets perpendicular to the $a$ axis; c) packing of these planes along the chain $b$ axis. Bottom: a) $\left[\mathrm{Gd}_{2}\right]$ building unit (only the first carbon of the benzene rings of N-BDC are 55 depicted) and b) its connection into a 3D framework in the structure of 2. $\mathrm{H}$ are omitted except those on water molecules, formate in a) and acetate in c). Colour code: Gd, purple; O, red; N, blue, C, black, H, beige.

Compound $1\left[\mathrm{Gd}(\mathrm{HCOO})(\mathrm{OAc})_{2}\left(\mathrm{H}_{2} \mathrm{O}\right)_{2}\right]^{\S}$ crystallizes in the monoclinic $P 2{ }_{1} / \mathrm{m}$ space group," the asymmetric unit containing a 60 a distorted square antiprismatic eight coordinate $\mathrm{Gd}^{3+}$ ion, two chelating terminal acetate, two aqua ligands, both in cis-position and all lying on one mirror plane, and a bridging $\mu-\mathrm{O}, \mathrm{O}$ ' formato ion lying on a parallel mirror plane. The structure of $\mathbf{1}$ thus builds on $1 \mathrm{D} \mathrm{Gd}^{3+}$ coordination chains propagating along the $b$ axis 65 through a single formato anti-anti bridge (Fig. 1a). Adjacent chains are rotated by $180^{\circ}$ with respect to $b$, so that the water molecules and acetato groups form a dense network of hydrogen bonds that results in thick sheets in the $b c$ plane (Fig. 1b and S1). The corresponding Gd $\cdots$ Gd separations are respectively $6.584(1)$ $70 \AA$ A through the -O-C-O- formate bridges and 5.920(1) A through the $-\mathrm{O}-\mathrm{H} \cdots \mathrm{O}-$ interchain $\mathrm{H}$-bonds. The sheets are separated by the acetate methyl groups (Fig. 1c), without any significant interactions, resulting in a inter-plane $\mathrm{Gd} \cdots \mathrm{Gd}$ separation of 7.997(1) $\AA$. The true topology of $\mathbf{1}$ is therefore likely better 
described as 2D. On the other hand, the structure of compound $\mathbf{2}^{£}$ is built from an edge-sharing $\left[\mathrm{Gd}_{2}\right]$ unit (Fig. 1d) connected through six N-BDC ligands in three dimensions to form a cubic porous framework with a $4^{12} \cdot 6^{3}$ topology (Fig. 1e), with Gd $\cdots \mathrm{Gd}$ 5 separations in the 10.488(2)-12.066(9) $\AA$ range. Within the dinuclear secondary building unit, the tricapped trigonalpyramidal $\mathrm{Gd}^{3+}$ ions are 4.073(6) $\AA$ apart, bridged through two $\mu$ $\mathrm{O}, \mathrm{O}$ '-carboxylato and two $\mu$-O,O,O'-carboxylato groups.

Figure S2 depicts the direct current (dc) susceptibilities 10 normalized per $\mathrm{Gd}^{3+}$ ion, which were collected for the $2-300 \mathrm{~K}$ temperature range in an applied field of $0.1 \mathrm{~T}$. For both compounds, the room-temperature $\chi^{(\mathrm{Gd})} T$ value is that expected for a spin-only $S=7 / 2 \mathrm{Gd}^{3+}$ ion $\left(7.875 \mathrm{~cm}^{3} \mathrm{Kmol}^{-1}\right)$. The value stays roughly constant as the temperature is decreased, and at 15 approximately $20 \mathrm{~K}$ the $\chi^{(\mathrm{Gd})} T$ decreases, reaching a minimum of approximately $7.1 \mathrm{~cm}^{3} \mathrm{Kmol}^{-1}\left(7.2 \mathrm{~cm}^{3} \mathrm{Kmol}^{-1}\right)$ at $2 \mathrm{~K}$, for 1 (2). In agreement with previous investigations, ${ }^{9}$ this behaviour points to very weak antiferromagnetic exchange for both complexes, being slightly weaker in the case of $\mathbf{2}$. Isothermal magnetizations 20 versus field are not particularly sensitive to slight differences in the exchange, therefore very similar plots (collected in the 2-10 $\mathrm{K}$ range and up to $5 \mathrm{~T}$, see Figure S3 in the Supporting Information) were observed for both complexes.

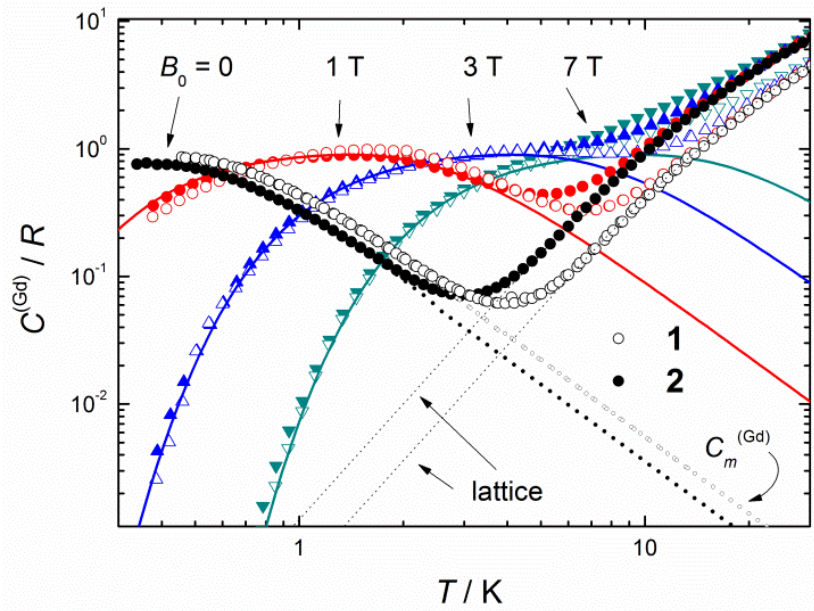

25 Fig. 2 Temperature-dependencies $(0.3-30 \mathrm{~K})$ of the heat capacity per $\mathrm{Gd}^{3+}$ ion $C^{(\mathrm{Gd})}$, normalized to the gas constant $R$ for $\mathbf{1}$ (empty markers) and 2 (filled markers) collected for $B_{0}=0,1,3$ and $7 \mathrm{~T}$, as labelled. Plotted also is the zero-field magnetic heat capacity $C_{m}{ }^{(\mathrm{Gd})}$ (small markers) and lattice contribution (dotted lines) for both complexes.

30 We next present the heat capacities $C^{(\mathrm{Gd})}$ of $\mathbf{1}$ and 2, which were collected in the $0.3-30 \mathrm{~K}$ range and up to $7 \mathrm{~T}$, and are depicted as normalized per $\mathrm{Gd}^{3+}$ ion in Figure 2. Close similarities between the measurements on the two compounds are easily discerned. At high temperatures, the heat capacity is 35 dominated by nonmagnetic contributions arising from thermal vibrations of the lattice. These can be modelled with a Debye function (dotted lines), obtaining the values for the Debye temperatures $\Theta_{\mathrm{D}}=80.4 \mathrm{~K}$ and $45.4 \mathrm{~K}$ for 1 and 2, respectively, evidencing a stiffer structure for the former (see, e.g., Fig. 1). The 40 low-temperature $C^{(\mathrm{Gd})}$ for $B_{0}>0$ is characterized by a fielddependent broad feature, arising from the splitting of the $S=7 / 2$ multiplet, which shifts to higher temperature by increasing $B_{0}$ (also calculated to provide the solid lines in Fig. 3). The zero- field $C^{(\mathrm{Gd})}$ presents a markedly different behaviour depending on 45 the compound examined, which we associate with the differences in the magnetic exchange. The very weak values of the exchange couplings that characterize these materials make it impossible to distinguish between the several exchange pathways, and only an average $J$ can be found. The estimate is obtained by comparing 50 the experimental $T^{-2}$ term, $C^{(\mathrm{Gd})} T^{2} / R=0.53 \mathrm{~K}^{2}$ and $0.36 \mathrm{~K}^{2}$, for 1 and 2, respectively, to the theoretical expression for the hightemperature "tail" of the magnetic heat capacity for Heisenberg coupling, i.e., $C^{(\mathrm{Gd})} T^{2} / R=6 z\left[S(S+1)|J| /\left(3 k_{\mathrm{B}}\right)\right]^{2}$, where $z$ is the number of nearest magnetic neighbours. ${ }^{12}$ This comparison 55 provides $z^{1 / 2} J / k_{\mathrm{B}}=-0.06 \mathrm{~K}$ and $-0.05 \mathrm{~K}$ for $\mathbf{1}$ and 2, respectively, that points to a weaker exchange for the latter in agreement with the susceptibility data (Fig. S2). From the heat capacity, the temperature-dependence of the magnetic entropy $S_{m}{ }^{(\mathrm{Gd})}$ is obtained by integration, using $S_{m}{ }^{(\mathrm{Gd})}(T)=\int C_{m}{ }^{(\mathrm{Gd})} / T \mathrm{~d} T$, where the 60 magnetic heat capacity $C_{m}^{(\mathrm{Gd})}$ is obtained from $C^{(\mathrm{Gd})}$ upon subtracting the lattice contribution. The thus-obtained $S_{m}{ }^{(\mathrm{Gd})}(T)$, depicted in Fig. S4 for the corresponding applied fields, tend to the maximum entropy value at high temperatures, corresponding to $R \ln (2 S+1)=2.08 R$, as proper for a $\mathrm{Gd}^{3+} S=7 / 2$ spin.
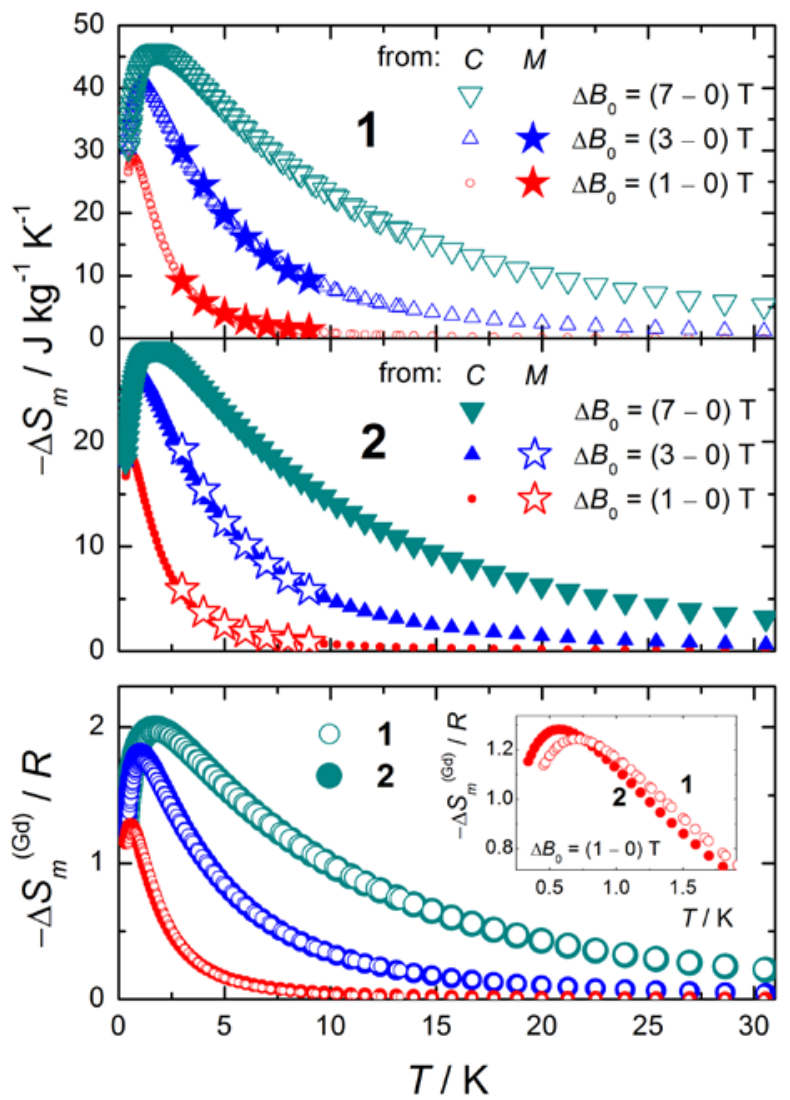

Fig. 3 Temperature-dependencies of the magnetic entropy change $\Delta S_{m}$, as obtained from magnetization $(M)$ and heat capacity $(C)$ data, for the indicated applied-field changes $\Delta B_{0}$, for 1 (top) and 2 (central). Bottom: entropy change per $\mathrm{Gd}^{3+}$ ion and normalized to $R$, as obtained from $C$, for

701 (empty markers) and 2 (filled markers). Inset: magnification of the lowest-temperature region and $\Delta B_{0}=1 \mathrm{~T}$.

To evaluate the MCE, we obtain the isothermal magnetic entropy change $\Delta S_{m}$, following a change in the applied magnetic field $\Delta B_{0}$, from the measured magnetization (Fig. S3) and heat 
capacity (Fig. 2), using known data-processing procedures. ${ }^{2,8}$ Both procedures provide identical results, depicted in Figure 3, proving the validity of our approach. It can be seen that $-\Delta S_{m}(T)$ reaches remarkable maxima, i.e., $45.9 \mathrm{Jkg}^{-1} \mathrm{~K}^{-1}$ for 1 and $29.0 \mathrm{Jkg}$ ${ }_{5}^{1} \mathrm{~K}^{-1}$ for 2 , both for $T=1.8 \mathrm{~K}$ and $\Delta B_{0}=(7-0) \mathrm{T}$. The difference mostly stems from the normalized molar weight per $\mathrm{Gd}^{3+}$ ion, i.e., $572.13 \mathrm{~g}$ for $\mathbf{1}$ and $366.38 \mathrm{~g}$ for $\mathbf{2}$. Indeed, both maximum values are close to the maximum available entropy per $\mathrm{Gd}^{3+}$, i.e. $2.08 R$. This is beautifully illustrated by the bottom panel of Fig. 3, 10 showing the set of entropy data already depicted in the top panels, although now normalized per $\mathrm{Gd}^{3+}$ ion and gas constant $R$. It is hardly possible to discern any difference between the behaviours of the two compounds, because the involved applied fields are largely overwhelming the effect due to the weak interactions 15 present. Only for the lowest field change $\Delta B_{0}=1 \mathrm{~T}$ (inset of Fig. 3 ), one can notice that $\mathbf{1}$ achieves a lower MCE, while being slightly shifted towards higher $T$, with respect to 2 , in agreement with the relatively stronger $J$ for $\mathbf{1}$, as it is well known that antiferromagnetic exchange does not favour the MCE. ${ }^{13}$

20 In summary, we report that the cryogenic MCE for Gd-based molecular refrigerant materials can be spectacularly large, regardless of the magnetic dimensionality. At liquid-helium temperatures and for the applied fields typically involved, the MCE is chiefly determined by the Gd density, that is the 25 metal:non-metal mass ratio. One can easily predict that this field will quickly move towards refrigerants with lighter ligands, because a larger MCE can be so achieved. We are still far from the ideal limit of an infinite ratio of non-interacting $\mathrm{Gd}^{3+}$ ions, for which the full entropy can be as large as $2.08 R / \mathrm{A}_{\mathrm{r}} \approx 110 \mathrm{Jkg}^{-1} \mathrm{~K}^{-1}$, 30 where $A_{r}$ is the relative $\mathrm{Gd}$ atomic mass. Our results open the field of cryogenic molecular coolers to extended frameworks, which will allow taking advantage of both the synthetic variety and intrinsic robustness of MOFs.

We are grateful to MINECO (contracts MAT2009-13977-C03, 35 MAT2011-24284 and CSD2007-00010), EC (for a Marie CurieIEF to GL), University of Granada (for a postdoctoral fellowship to MAP), and EPRSC for funding.

\section{Notes and referencies}

${ }^{a}$ Instituto de Ciencia de Materiales de Aragón, CSIC-Universidad de 40 Zaragoza, Departamento de Física de la Materia Condensada, 50009 Zaragoza (Spain). E-mail: roubeau@unizar.es; evange@unizar.es; WWW: http://molchip.unizar.es/.

${ }^{b}$ EaStCHEM School of Chemistry, The University of Edinburgh, West Mains Road, Edinburgh, EH93JJ (UK)

$45 \dagger$ Electronic Supplementary Information (ESI) available: [selected bond distance and angles for $\mathbf{1}$, crystallographic data in CIF format, synthesis and characterization of 2, isothermal magnetizations for $\mathbf{1}$ and 2]. See DOI: $10.1039 / \mathrm{b} 000000 \mathrm{x} /$

$\S$ Synthesis of $1 . \mathrm{Gd}(\mathrm{OAc})_{3} \cdot 6 \mathrm{H}_{2} \mathrm{O}(442 \mathrm{mg}, 1 \mathrm{mmol}), \mathrm{HCOOH}(40 \mu \mathrm{l}, 1$ $50 \mathrm{mmol})$ and $\mathrm{Et}_{3} \mathrm{~N}(140 \mu \mathrm{l}, 1 \mathrm{mmol})$ were dissolved in $25 \mathrm{ml}$ of $\mathrm{CH}_{3} \mathrm{CN}$ and stirred for two hours. The resulting white powder was filtered, washed with acetonitrile and dried. Recrystallisation from $\mathrm{H}_{2} \mathrm{O}$ affords crystals of 1 in $\sim 22 \%$ yield after $\sim 40$ days. Elemental analysis (\%) calculated for $\mathrm{C}_{5} \mathrm{H}_{11} \mathrm{O}_{8} \mathrm{Gd}$ : C 16.85, H 3.11; found: C 16.71, H 3.23.

55 " Crystal data for 1: data were obtained on an Agilent XCalibur diffractometer at $100 \mathrm{~K}$ from Mo $K \alpha(\lambda=0.71073)$ on a colourless plate, monoclinic, space group $P 22_{1} / \mathrm{m}$ (no. 11), with $a=7.9966(4) \AA, b=$ 6.5839(2) $\AA, c=9.9447(4) \AA, \beta=109.425(5)^{\circ}, V=493.77(4) \AA^{3}, Z=2$, $\rho_{\text {calcd }}=2.397 \mathrm{~g} / \mathrm{cm}^{3}, \mu=6.734 \mathrm{~mm}^{-1} .4816$ reflections were measured, 601283 of which were independent. Refinement converged at final $\mathrm{w} R 2=$
0.0758, $R 1=0.0302$ and $S=1.079$ (for 1095 reflections with $I>2 \sigma(I)$ ). CCDC 881702.

$£$ Compound 2 was prepared as in ref. 9 and analyzed satisfactorily.

Magnetic measurements were performed using a commercial SQUID 65 magnetometer. The measured values were corrected for the experimentally measured contribution of the sample holder, while the derived susceptibilities were corrected for the diamagnetism of the samples, estimated from Pascal's tables. Heat capacities in the range 0.35$130 \mathrm{~K}$ were obtained using the relaxation method in a commercial ${ }^{3} \mathrm{He}$ 70 set-up, equipped with a $7 \mathrm{~T}$ magnet. The samples were in the form of microcrystalline powder, mixed with Apiezon- $\mathrm{N}$ grease to provide good internal thermal contact between crystallites, heater, and thermometer.

1 W. F. Giauque, J. Am. Chem. Soc., 1927, 49, 1864.

2 M. Evangelisti, F. Luis, L. J. de Jongh and M. Affronte, J. Mater. 75 Chem., 2006, 16, 2534; M. Evangelisti and E. K. Brechin, Dalton Trans., 2010, 39, 4672.

3 R. Sessoli, Angew. Chem. Int.-Ed., 2012, 51, 43.

4 G. Karotsis, M. Evangelisti, S. J. Dalgarno and E. K. Brechin, Angew. Chem. Int. Ed., 2009, 48, 9928; G. Karotsis, S. Kennedy, S. J.

80 Teat, C. M. Beavers, D. A. Fowler, J. J. Morales, M. Evangelisti, S. J. Dalgarno and E. K. Brechin, J. Am. Chem. Soc., 2010, 132, 12983; J. B. Peng, Q. C. Zhang, X. J. Kong, Y. P. Ren, L. S. Long, R. B. Huang, L. S. Zheng and Z. P. Zheng, Angew. Chem. Int. Ed., 2011, 50, 10649; M. Evangelisti, O. Roubeau, E. Palacios, A. Camón, T. N. Hooper, E. K. Brechin and J. J. Alonso, Angew. Chem. Int. Ed., 2011, 50, 6606; J. W. Sharples, Y. Z. Zheng, F. Tuna, E. J. McInnes and D. Collison, Chem. Commun., 2011, 47, 7650; Y. Z. Zheng, M. Evangelisti and R. E. P. Winpenny, Chem. Sci., 2011, 2, 99; S. K. Langley, N. F. Chilton, B. Moubaraki, T. Hooper, E. K. Brechin, M. Evangelisti and K. S. Murray, Chem. Sci., 2011, 2, 1166; Y. Z. Zheng, M. Evangelisti and R. E. P. Winpenny, Angew. Chem. Int. Ed., 2011, 50, 3692; A. Hosoi, Y. Yukawa, S. Igarashi, S. J. Teat, O. Roubeau, M. Evangelisti, E. Cremades, E. Ruiz, L. A. Barrios and G. Aromí, Chem. Eur. J., 2011, 17, 8264; Y. Z. Zheng, M. Evangelisti, $95 \quad$ F. Tuna and R. E. P. Winpenny, J. Am. Chem. Soc., 2012, 134, 1057; T. Birk, K. S. Pedersen, C. Aa. Thuesen, T. Weyhermüller, M. Schau-Magnussen, S. Piligkos, H. Weihe, S. Mossin, M. Evangelisti and J. Bendix, Inorg. Chem., 2012, 51, 5435.

5 F. S. Guo, J. D. Leng, J. L. Liu, Z. S. Meng and M. L. Tong, Inorg. $100 \quad$ Chem., 2012, 51, 405.

6 L. Sedláková, J. Hanko, A. Orendáčová, M. Orendáč, C. L. Zhou, W. H. Zhu, B. W. Wang, Z. M. Wang and S. Gao, J. Alloys Compd., 2009, 487, 425.

7 M.-J. Martínez-Pérez, O. Montero, M. Evangelisti, F. Luis, J. Sesé, S. 105 Cardona-Serra and E. Coronado, Adv. Mater., 2012, to be published.

8 V. K. Pecharsky and K. A. Gschneidner Jr., J. Magn. Magn. Mater., 1999, 200, 44.

9 J. Sánchez Costa, P. Gamez, C. A. Black, O. Roubeau, S. J. Teat and J. Reedijk, Eur. J. Inorg. Chem., 2008, 1551; C. A. Black, J. Sánchez Costa, W. T. Fu, C. Massera, O. Roubeau, S. J. Teat, G. Aromí, P. Gamez and J. Reedijk, Inorg. Chem., 2009, 48, 1062.

10 N. Stock and S. Biswas, Chem. Rev., 2012, 112, 933.

11 E. Manuel, M. Evangelisti, M. Affronte, M. Okubo, C. Train and M. Verdaguer, Phys. Rev. B, 2006, 73, 172406; M. Evangelisti, E. Manuel, M. Affronte, M. Okubo, C. Train and M. Verdaguer, J. Magn. Magn. Mater., 2007, 316, e569.

12 A. Abragam and B. Bleaney, Electron Paramagnetic Resonance of Transition Ions, (Oxford University Press, Oxford, England, 1970).

13 T. N. Hooper, J. Schnack, S. Piligkos, M. Evangelisti and E. K. 120 Brechin, Angew. Chem. Int. Ed., 2012, 51, 4633. 